\title{
Early trial results favor surgery over stenting for symptomatic carotid artery stenosis
}

| nterim results of the International Carotid Stenting Study (ICSS), now published in The Lancet, suggest that endarterectomy represents a safer treatment option than stenting for patients with symptomatic carotid artery stenosis. According to these results, the difference in safety observed between the two procedures largely stemmed from a higher rate of post-treatment nondisabling strokes in the stenting group.

Stenting, with or without protection devices, has been widely adopted as an alternative to endarterectomy in the treatment of symptomatic carotid artery stenosis. Nevertheless, although endovascular procedures have been shown to circumvent the main complications associated with such surgery (cranial nerve injury and hematoma), randomized trials have failed to demonstrate that stenting shows higher efficacy than endarterectomy or achieves a comparable overall level of safety.

The primary aim of the ICSS, a multicenter randomized controlled trial, is to assess the 3 year rate of fatal or nondisabling stroke in individuals with symptomatic carotid stenosis following treatment with endarterectomy or stenting. Data relating to this aim are not yet available. The current report details the rates of mortality and morbidity associated with these procedures at 120 days postrandomization (the intention-to-treat analysis) and 30 days after treatment (per-protocol analysis).

In total, 1,713 patients with recently symptomatic stenosis were randomly allocated to endarterectomy $(n=858)$ or stenting $(n=855)$ groups, with most of these patients undergoing their designated treatment (821 individuals in the surgery group versus 828 individuals in the stenting cohort).

In the intention-to-treat analysis, the incidence of stroke, death or procedural myocardial infarction was found to be $5.2 \%$ (44 events) in patients who underwent

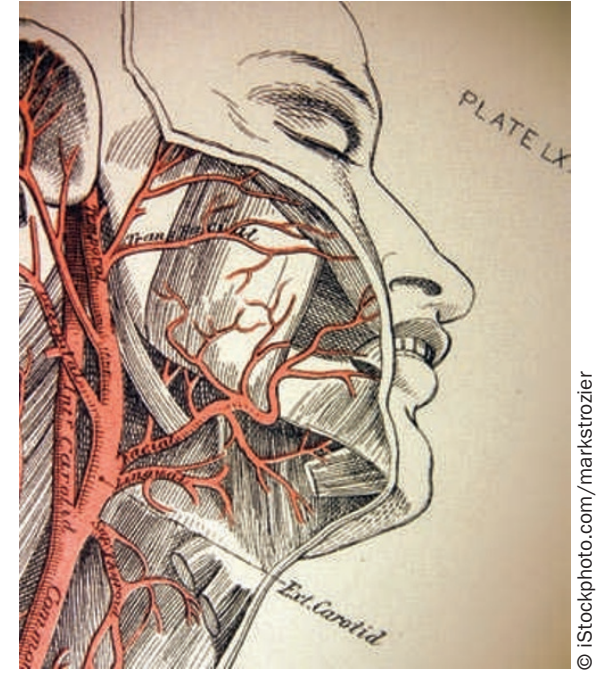

surgery and $8.5 \%$ (72 events) in individuals who were treated with stenting. Similar results were reported from the per-protocol analysis, with the rate of stroke, death or myocardial infarction rising from $4 \%(33$ events) in the endarterectomy group to $7.4 \%$ (61 events) in the stenting cohort. A breakdown of the figures from both analyses revealed that the differences in the rates of events were largely due to the number of nondisabling strokes that occurred in each group. For example, according to the figures from the perprotocol analysis, 36 nondisabling strokes were reported in the stenting group while 11 such events were noted in individuals who underwent surgery. By contrast, the results from this analysis indicated that 14 disabling strokes occurred in each group.

In addition to a difference in the rates of nondisabling stroke, both analyses showed that the number of fatal strokes or mycocardial infarctions was higher with stenting than with surgery. The perprotocol analysis revealed that 11 such events occurred in the stenting group, whereas only 3 events of this type occurred after surgery. The overall rates of disabling stroke or death following both procedures, however, were not markedly different in each analysis.
The clinicians involved in the 30 day follow-up, while not being involved in the randomization process, were not blinded to the treatment each patient received. To address the concern of potential ascertainment bias of events such as nondisabling stroke, an MRI substudy was conducted on subsets of patients randomly assigned to undergo stenting or surgery. The primary outcome measure of the study was the appearance of at least one new ischemic lesion on diffusion-weighted imaging on the post-treatment scan (conducted 1-3 days after treatment).

According to the MRI substudy, the results of which are now published in The Lancet Neurology, 50\% of the 124 patients who underwent stenting exhibited at least one new ischemic lesion on the post-treatment scan. By contrast, only $17 \%$ of the 107 patients in the surgery group developed one or more such lesions following treatment. The researchers conclude that the increased risk of stroke observed with stenting was probably not the result of ascertainment bias.

According to the ICSS investigators, the data from their interim analysis are in line with results from previous randomized controlled trials of endarerectomy versus stenting in the treatment of symptomatic carotid stenosis and suggest that, for now, endarerectomy should remain the preferred treatment for this condition in patients who are suitable for surgery. Nevertheless, the researchers stress that data from the 3 year follow-up are required to determine the long-term efficacy of stenting compared with endarerectomy.

\section{Darran Yates}

\footnotetext{
Original articles International Carotid Stenting Study Investigators. Carotid artery stenting compared with endarterectomy in patients with symptomatic carotid stenosis (International Carotid Stenting Study): an interim analysis of a randomised controlled trial. Lancet 375, 985-997 (2010) | Bonati, L. H. et al. New ischaemic brain lesions on MRI after stenting or endarterectomy for symptomatic carotid stenosis: a substudy of the International Carotid Stenting Study (ICSS) Lancet Neurol. 9, 353-362 (2010)
} 